\title{
Aerobic exercise ameliorates learning and memory deficits of aging rats induced by D-galactose via promoting SYP and BNDF expression in hippocampus
}

\author{
Yan $\mathrm{Fu}^{1,2}$, Yeting Zhang ${ }^{3}$ and Qiongjia Yuan ${ }^{2, a}$ \\ ${ }^{1}$ Department of Physical Education, Southwest University for Nationalities, Chengdu, China \\ ${ }^{2}$ Department of Sports Medicine, Chengdu Sport University, Chengdu, China \\ ${ }^{3}$ Department of Physical Education, Chengdu University, Chengdu, China
}

\begin{abstract}
The aim of this study was to explore the effects of aerobic exercise on learning and memory of aging brain and the underlying potential mechanism. We adopted the method of intraperitoneal administration for 6 weeks to induce aging rat model and gave rats swimming exercise intervention in the process of aging modeling. Then the Morris Water Maze test, immunofluorescence, western blotting and real time PCR technology were adopted to estimate rats' learning and memory abilities and the expression levels of BDNF and SYP in hippocampus respectively. Compared with the aging model rats induced by D-gal administration, the rats subjected to swimming exercise in the process of aging modeling showed not only faster acquisition and better retention of the maze but also higher expression levels of BDNF and SYP in hippocampus. These findings provide evidence that aerobic exercise could ameliorate learning and memory deficits of aging rats induced by D-gal, which is related to aerobic exercise' promoting hippocampal BNDF and SYP expression.
\end{abstract}

\section{Introduction}

The decline in cognitive functions, especially learning and memory deficits, is prevalent among older adults, which results in degraded quality of life for them[1,2]. Therefore, how to slow down the impairments of learning and memory in the aging process to promote the development of cognitive function is one of the goals of public health. It has been demonstrated by both epidemiological and experimental studies that physical exercises contribute to achieving the goal[3,4], however, the underlying mechanism remains incompletely unclear.

It is well known that the learning and memory formation are based on the neuron synaptic plasticity[5]. Synaptophysin(SYP), one of the most widely used protein markers of synaptic plasticity in the brain, existing in almost all pre-synaptic membranes and vesicles, is thought essential for stimulating synapse formation and synaptic transmission process[6,7]. Previous studies have confirmed that the decrease of SYP in hippocampus, an important brain area responsible for learning and memory, is relevant to age-dependent impairments in learning and memory[8,9]. Whether aerobic exercise has influence on SYP in aging hippocampus still remains unknown.

Brain-derived neurotrophic factor (BDNF) plays a critical role in the survival, growth, differentiation and synaptic plasticity of mammalian neurons[10,11]. It is well documented that low

\footnotetext{
${ }^{a}$ Corresponding author:yqj1225@163.com

This work was supported by grants from the National Natural Science Foundation of China (No.31371202), the Scientific research project of Sichuan Education Department (No. 15ZA0294),
} 
BDNF expression in brain involved in the learning and memory deficits in the process of aging [12]. Available data in the literature indicate that regular aerobic exercise may promote BDNF release in budding and adult hippocampus[13-15], but few experiments focus on the effects of exercises on BDNF expression in aging hippocampus.

The free radical theory of aging indicates that increase of free radicals and oxidative stress and decline of anti-oxidation function are the main factors leading to aging and even to shortening lifespan[16]. D-galactose (D-gal), a reducing sugar, is a naturally occurring metabolic substance in body. At high levels, it can result in the excessive production of oxygen free radicals that damage cellular lipids, proteins, and DNA, which eventually impairs normal function of macromolecules and cells[17]. It has been shown that prolonged systemic exposure of rodents to D-gal makes oxidative stress increase and anti-oxidation function decline, which causes brain cell biological membrane peroxide reaction, and finally results in degenerative changes of brain[17,18]. These changes are considerably similar to the symptoms in natural aging brain. Thus, the aging rodent model induced by D-gal is recognized as an ideal model for studying the mechanism underlying brain aging and the approach for anti-aging.

In the study, we have used the aging rat model induced by d-gal to verify directly the hypothesis that regular aerobic exercise intervention would ameliorate learning and memory deficits of aging rats via promoting SYP and BNDF expression in hippocampus.

\section{Materials and methods}

\subsection{Animals and groups}

Clean grade healthy adult male Sprague-Dawley rats, each weighing 280-320g, were purchased from the experimental animal center of Chengdu Dasuo Biological Technology Co. LTD(Chengdu, China; Animal production license: CSXK, 2012-24). All rats were kept in conditions of temperature (22$25^{\circ} \mathrm{C}$ ) and humidity $(45-65 \%)$ and $12 \mathrm{~h} / 12 \mathrm{~h}$ light-dark cycle, with food and water available ad libitum. After acclimatization to the laboratory for 1 week, the rats were randomly divided into 3 groups: control(C) group, D-gal administration (D) group, D-gal administration with exercise (DE) group, 12 rats in each group. All procedures were performed in accordance with Animal Care and Use Guidelines in China.

\subsection{D-gal administration}

According to literature published[19], the study adopted the method of systemic injection of D-gal (sigma, USA) to establish aging rat model, with rats in group D and DE intraperitoneally injected withD-gal at dose of $100 \mathrm{mg} / \mathrm{kg}$. d (dissolved in $0.9 \%$ saline to $5 \%$ concentration, injection volume was $2 \mathrm{ml} / \mathrm{kg}$. d) daily for 6 weeks to induce aging, whereas those in group C were intraperitoneally injected with $0.9 \%$ saline in the same volume with D-gal.

\subsection{Exercise protocol}

During the 6 weeks of D-gal administration, the rats in group DE were subjected to exercise intervention. The endurance swimming exercise training in a transparent glass tank $(150 \mathrm{~cm}$ in length, $60 \mathrm{~cm}$ in width, $50 \mathrm{~cm}$ in depth, with the water temperature kept at $33 \pm 1^{\circ} \mathrm{C}$ ) was used in our study[19]. On the first day of training, the rats swam for $20 \mathrm{~min}$, and then the training duration was lengthened daily by $10 \mathrm{~min}$ until each rat could swim continuously for $60 \mathrm{~min}$. Subsequently, rats swam $1 \mathrm{~h}$ per day for 6 times a week (9:00-10:00 PM from Monday to Saturday). 


\subsection{Morris Water Maze (MWM) test}

The MWM test, consisting of 6 consecutive days of oriented navigation trial and a $60 \mathrm{~s}$ of probe trial, was performed to evaluate spatial learning and memory as described previously by Morris[20] with some modifications. The water maze was a black circular pool $(150 \mathrm{~cm}$ in diameter, $50 \mathrm{~cm}$ in height) filled with opaque water at $23 \pm 1^{\circ} \mathrm{C}$ and surrounded by various visual cues. Moreover, the tank was divided into 4 equal quadrants (marked respectively as $\mathrm{E}, \mathrm{S}, \mathrm{W}$ and $\mathrm{N}$ ) with two imaginary perpendicular lines. During the oriented navigation trial, each rat participated in 4 trials per day to learn to locate the platform $(9 \mathrm{~cm}$ in diameter) that fixed in the quadrant $\mathrm{E}$ and submerged $1.5 \mathrm{~cm}$ underneath the surface of water. The animal was gently placed into the pool at the midpoint of the edge of quadrant, changed for each trial, and was allowed a maximum of 120 s to locate the platform. Rats failing to find the platform within $120 \mathrm{~s}$ were guided towards it and remained there for $15 \mathrm{~s}$, the same period as was allowed for the successful animals. The time that it took the rat to arrive at the submerged platform (referred to as "escape latency") and the swimming speed were recorded with a digital camera to evaluate spatial learning ability. $24 \mathrm{hrs}$ after the last navigation training trial, the platform was removed and each rat was subjected to the probe trial, during which the rats were placed into the tank at the midpoint of the edge of quadrant $\mathrm{W}$ and allowed to swim in the tank for $60 \mathrm{~s}$. The time spent in the target quadrant (quadrant E) and the time of crossing the location where the platform had been fixed were recorded as a measure of spatial memory.

\subsection{Tissue sampling}

Tissue samples were collected in the $24 \mathrm{hrs}$ after the MWM test, with all rats sacrificed by decapitation and the brains quickly removed from the skull. 6 whole brain samples per group were immersed in $4 \%$ paraformaldehyde for immunofluorescence assay, while the bilateral hippocampus of the other 6 brains were quickly dissected on ice and stored at $-80^{\circ} \mathrm{C}$ for western blot and real-time PCR analysis.

\subsection{Brain section and immunofluorescence assay for SYP}

The brain tissue samples fixed in $4 \%$ paraformaldehyde were embedded in paraffin and coronally sectioned at $50 \mu \mathrm{m}$ thickness using a microtome (Leica, Wetzlar, Germany), and five tissue sections were selected at $30 \mu \mathrm{m}$ apart between +1.7 and $+3.0 \mathrm{~mm}$ posterior to the bregma referring to a mouse atlas[21]. SYP immunofluorescence staining was performed in accordance with the standard protocols. DAPI was used for nuclear staining. In the end, $20 \mu 1$ of glycerole was applied to each slide and a cover slip was sealed in place. In order to obtain accurate data, immunofluorescence staining was carried out carefully under the same conditions and fluorescence images were obtained by the OLYMPUS microscope and analyzed by using Image-Pro Plus 6.0 software. SYP immunoreactivity was evaluated by measuring the integral optical density (IOD) of SYP immuno positive contents.

\subsection{Protein extraction and western blot analysis for SYP and BDNF}

The right hippocampus samples stored at $-80^{\circ} \mathrm{C}$ were used for BDNF and SYP protein quantification using western blot. Total protein in the hippocampus was extracted as previously described[19]. Protein content was measured by BCA assay (Thermo Scientific) according to the standard protocols. Sample containing $50 \mu \mathrm{g}$ proteins was electrophoresed on 5-20\% gradient SDS-PAGE gels to separate and then blotted onto low-fluorescent polyvinylidene fluoride (PVDF) membranes. After having been blocked with 5\% non-fat milk for $1 \mathrm{~h}$, the PVDF membrane was incubated overnight at $4{ }^{\circ} \mathrm{C}$ with the anti-BDNF or anti-SYP antibody(both are polyclonal rabbit anti-rat antibody, diluted at 1:500, Santa Cruz, USA) and then incubated for another $2 \mathrm{hrs}$ with appropriate horseradish peroxidase-conjugated secondary antibody(polyclonal goat anti-rabbit $\mathrm{IgG}$, diluted at 1:2000, Santa Cruz, USA). The membranes were visualized by using the enhanced chemiluminescence detection system (Pierce,USA). $\beta$-actin (polyclonal, diluted at 1:5000; Abcam, Cambridge, MA) was used for internal control. Integral 
optical density (IOD) of immunoblots was quantified by using Quantity One Gel Imaging System (Bio Rad, USA) and was normalized to $\beta$ - actin.

\subsection{RNA Extraction and Real-Time PCR Analysis for BDNF mRNA}

Remaining hippocampus samples stored at $-80^{\circ} \mathrm{C}$ were used for BDNF mRNA using real-time PCR analysis. Total RNA in hippocampus was extracted using TRIZOL Reagent (TaKaRA, Japan). After removing genomic DNA residual using DNase I, RNA samples were reversely transcribed into complementary DNA (cDNA) using the iScript ${ }^{\mathrm{TM}}$ cDNA Synthesis Kit(BIO-RAD,USA)in accordance with the manufacturer's protocols. Then the real-time PCR was carried out using GoTaq ${ }^{\circledR}$ qPCR Master Mix (Promega, USA) and CFX96 Touch ${ }^{\mathrm{TM}}$ Real-Time PCR Detection System (Bio-Rad, USA). After an initial incubating at $94^{\circ} \mathrm{C}$ for $10 \mathrm{~min}$, DNA samples were amplified for 40 cycles of $95^{\circ} \mathrm{C}$ denaturation for $15 \mathrm{~s}$, annealing at $60^{\circ} \mathrm{C}$ for $60 \mathrm{~s}, 72^{\circ} \mathrm{C}$ extension for $1 \mathrm{~min}$. For each sample, the levels of BDNF mRNA were normalized against $\beta$-actin mRNA levels using the $2^{-\Delta \Delta C t} \operatorname{method}[22]$. The sequences of primers for RT-PCR amplification were as follows: BDNF(forward primer: $5^{\prime}$-AGC GTG TGT GAC AGT ATT AGC GAG-3' and reverse primer: 5'-ATG GGA TTA CAC TTG GTC TCG TAG A-3'), $\beta$-actin(forward primer: 5'-CGT AAA GAC CTC TAT GCC AAC A-3' and reverse primer: 5'-TAG GAG CCA GGG CAG TAA TC-3').

\subsection{Data analysis}

All values were reported as the means \pm standard deviation $(\mathrm{M} \pm \mathrm{SD})$. In terms of the data from the probe trials, western blot analysis and real-time PCR were made using a one-way analysis of variance (ANOVA) by SPSS (SPSS Inc, Chicago, IL, USA) 19.0 for windows, while the data from oriented navigation trial was analysed with a two-way ANOVA. A value of $P<0.05$ was considered statistically significant.

\section{Results}

\subsection{Effects of aerobic exercise on spatial learning and memory ability}

In the MWM navigation task, all animals' escape latencies gradually shortened (Figure 1a), indicating that each rat was able to learn to locate the submerged platform. The rats in the group $\mathrm{C}$ and $\mathrm{DE}$ showed maximal performance on day 3 and did not significantly improve thereafter, whereas those in group D improved at a lower pace, and reached maximal performance on day 5(Figure 1a). Analysis of the average escape latencies demonstrates that the group D were significantly longer than the group $\mathrm{C}$ and DE on days 2,3 , and 4 of training $(p<0.05)$, while there was no statistical difference on days 1 , 5 and 6 among these groups as regards $(p>0.05)$. There was no significant difference in swimming speed among 3 groups during the 6-day training period (Figure 1b), suggesting that the escape latencies were not influenced by swimming velocity. In the probe trial, the rats in group D spent less time in the target quadrant compared with both group $\mathrm{C}$ and $\mathrm{DE}(p<0.05)$, however, there was no remarkable difference in that between group DE and C (Figure 1c). In addition, rats in group C and $\mathrm{DE}$ crossed where the platform was fixed more often than group D $(p<0.05)$ (Figure $1 \mathrm{~b})$. Taken together, these data suggested that D-gal administration of 6 consecutive weeks impaired spatial learning and memory, while the aerobic exercise ameliorated the learning and memory impairment caused by D-gal administration. 

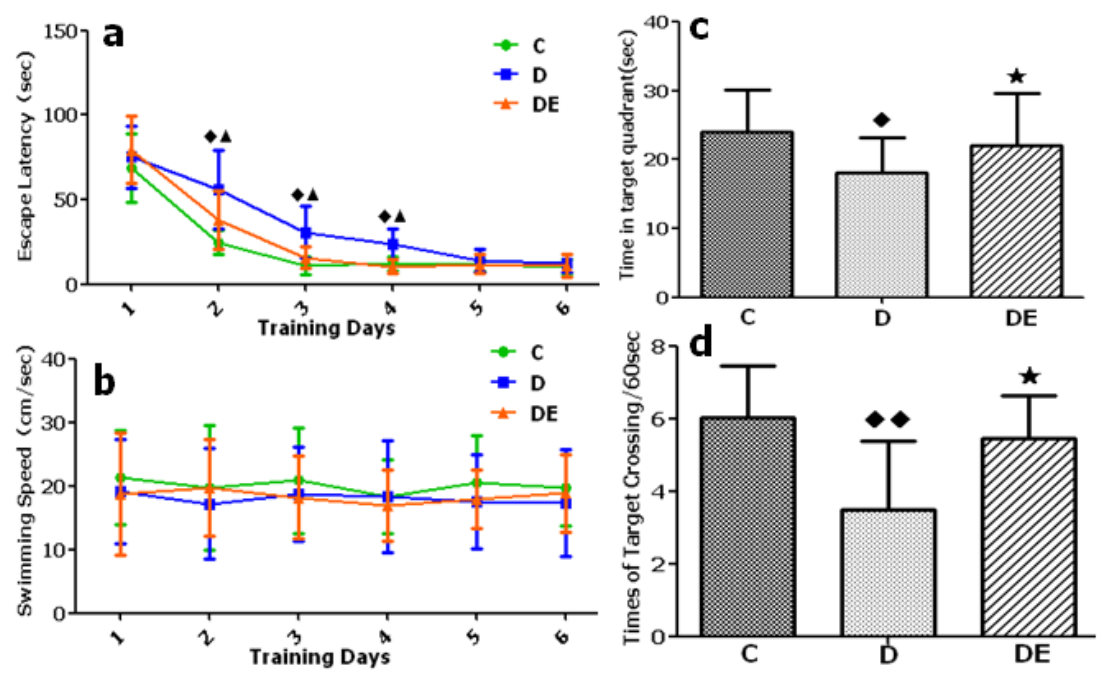

Figure 1. Changes in spatial learning and memory using the MWM test.

(a) Escape latency on each training day. (b) Mean swimming speed on each training day.( c) Number of crossings platform in the target quadrant during 60 seconds probe trail. (d) The time spent in the target quadrant during probe trial. $\diamond P<0.05, \diamond \diamond P<0.01, v s$. the group C; $\Delta P<0.05$, vs. the group DE; $\star P<0.05$, vs. the group D.

\subsection{Effects of Aerobic Exercise on SYP Protein Expression in Hippocampus}

Under the laser confocal microscope, the immune positive products of SYP were observed to scattered distribution in hippocampus, and compared with group D, the fluorescence intensities of group C and group DE were more intense (Figure 2). Further analysis indicated that the IOD value of SYP immunofluorescence positive products in group $\mathrm{D}$ was lower than group $\mathrm{C}(\mathrm{P}<0.01)$, while that of group DE was significantly higher than group $\mathrm{D}(\mathrm{P}<0.01)$ (Figure $3 \mathrm{a})$.

Consistent with the results of immunofluorescence assay, western blot analysis revealed that SYP protein in group D was significantly lower than both group $\mathrm{C}(P<0.01)$ and DE $(p>0.05)$. There was no statistical difference between the group $\mathrm{C}$ and $\mathrm{DE}$. The present results show that chronic administration D-gal caused the SYP expression levels in hippocampus decline, while exercise may upregulate SYP, a marker for synaptic plasticity in hippocampus thus reversed the downward to some extent caused by the D-gal treated (Figure $3 b$ ).
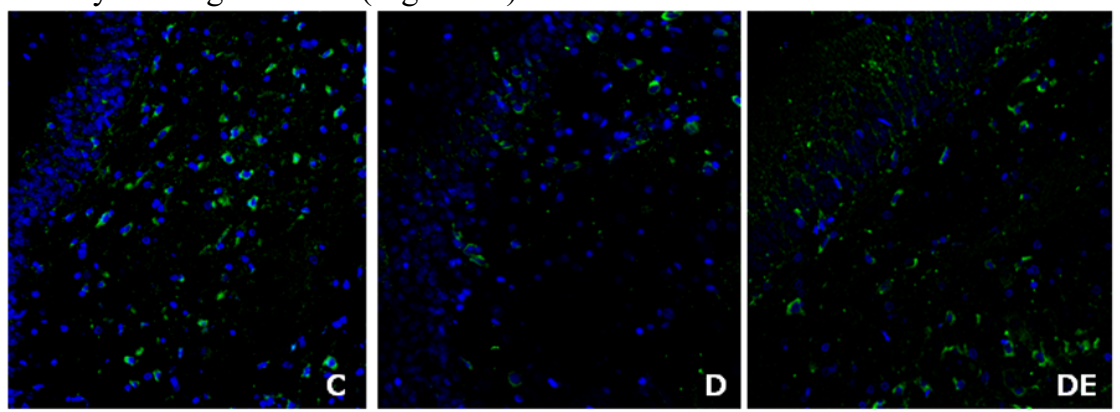

Figure 2. Immunofluorescence micrographs of SYP in hippocampus.

Note: Blue-fluorescence: nucleus tagged by DAPI; Green fluorescence: SYP tagged by FITC. 

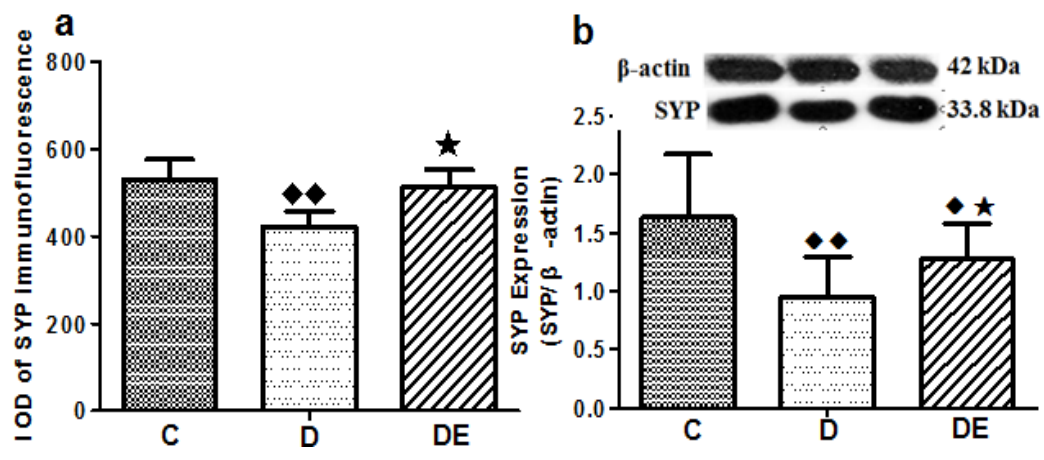

Figure 3 Changes in hippocampal SYP protein.

(a) IOD of SYP immunofluorescence positive products in hippocampus. (b) SYP protein levels in hippocampus using western blot. $\diamond P<0.05, \diamond \diamond P<0.01, v s$. the group $\mathrm{C} ; \star P<0.05$, vs. the group D.

\subsection{Effects of Aerobic Exercise on BDNF protein and mRNA Levels in Hippocampus}

According to Western blot analysis, the rats in group $\mathrm{D}$ had obviously less BDNF level in hippocampus than group $\mathrm{C}$ and $\mathrm{DE}(\mathrm{P}<0.01, \mathrm{P}<0.05$; Figure $4 \mathrm{a})$, and that the $\mathrm{BDNF}$ levels slightly declined in group $\mathrm{DE}$ compared with group $\mathrm{C}$, however, these values did not reach statistical significance $(p>0.05$; Figure $4 a)$. Consistent with those of the west blot analysis, the results from realtime PCR showed that the levels of hippocampal BDNF mRNA were significantly declined in the group D compared with both group $C$ and group $\mathrm{DE}$ ( $\mathrm{p}>0.05$; Figure $4 \mathrm{~b})$. These findings demonstrated that D-gal administration restrains BDNF genetic transcription as well as protein synthesis, however, exercise intervention attenuated the inhibitions induced by D-gal administration.
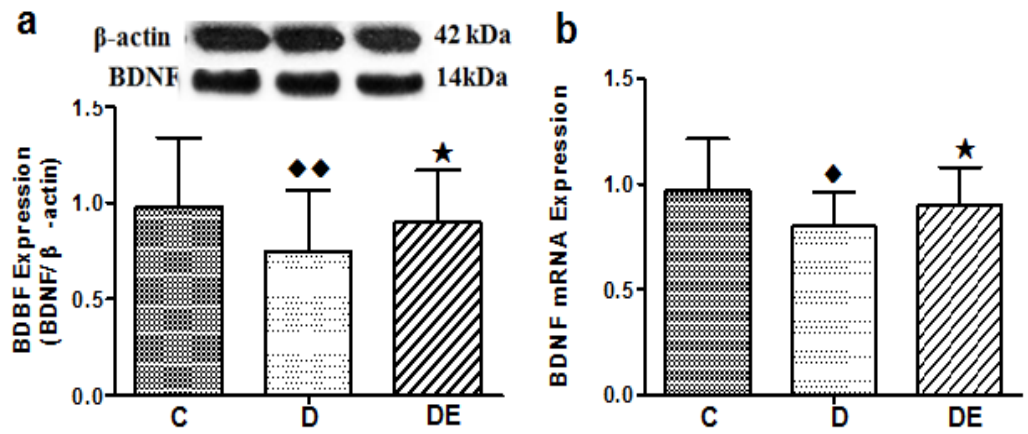

Figure 4 Changes in hippocampal BDNF protein and mRNA.

(a) BDNF protein levels in hippocampus. (b) BDNF mRNA levels in hippocampus. $\diamond P<0.05, \diamond \diamond P<0.01, v s$. the group $\mathrm{C} ; \star P<0.05$, vs. the group D.

\section{Discussion}

Nowadays, systemic D-gal administration is one of the most extensively used brain aging model building methods[17, 18]. Our previous studies have confirmed that rats injected with D-gal for 6 weeks resulted in deterioration of learning and memory capacity and increase of free radicals in the brain[19], which is similar to the performance in natural aging. In order to test the influence of aerobic exercise on the hippocampal SYP and BDNF of aging rats' and to explore the effects of aerobic exercise on learning and memory of aging brain and the potential mechanism underlying, we built the aging model in this way again and gave rats swimming exercise intervention in the process of aging modeling in the current study. 
It is generally acknowledged that synapse is the key structure of neuron networking and information transmission and that neuronal synaptic plasticity is the important part of neurobiology for learning and memory ability[10,11]. Many studies have implicated a contribution of synaptic plasticity reduction in learning and memory dysfunction in the aging process[23, 24]. SYP, a membrane protein with $38 \mathrm{kD}$ molecular weight, distributes nearly in all pre-synaptic terminals and is involved in synapse formation, neurotransmitter release and synaptic vesicle cycle[6,7], etc. At present, SYP is recognized as a reliable marker protein for synaptic plasticity in the brain and often used to qualitatively or quantitatively analyze synaptic density and distribution[6]. It has been shown that SYP levels in hippocampus decline with aging and present an age-dependent trend[25]. In the aging model induced by D-gal in our study, the hippocampal SYP expression is also down-regulated, suggesting that synapse degenerative changes in hippocampus, which may be an important cause of learning and memory impairment induced by D-gal treated.

Despite the fact that there is no way to stop the physiological brain aging process, yet numerous epidemiological surveys and experimental studies indicate that appropriate sports could delay the process of aging brain and improve the learning and memory ability [3, 4]. Consistent with what has been previously reported[19], rats' performance in MWM demonstrates that regular swimming exercise could prevent learning and memory impairment against brain senescence in the present study, and it is possible that this prevention effect for learning and memory impairment may be a consequence of improved synaptic plasticity and neurotransmission through the increase of SYP expression. This point is based on the observation that both immunofluorescence and western blot assay of hippocampal SYP in the present study showed significantly higher expression level in rats that did exercise in the process of D-gal injection than rats that didn't exercise.

BDNF, one of the most abundant neurotrophin factors, widely distributing in the central nervous system, has been confirmed by animal studies that it could regulate hippocampal synaptic plasticity and cognitive function through multiple mechanisms[26,27], and its' expression level in hippocampus is closely related to the ability of learning and memory[10,11]. BDNF knockout mice exhibit decreases in the number of docked vesicles and in the levels of SYP and impairments in neurotransmitter release[28]. A lot of experimental results show that BDNF amount in brain, especially in hippocampus, is reduced in the aged[12,29,30]. Consistent with these results, we found that both BDNF mRNA and BDNF protein levels in hippocampus of aging model rats were significantly lower than the adult rats in the current study. Exercise has been found to increase hippocampal BDNF both in the growth period and adulthood. In the present study, we investigated the effect of aerobic exercise intervention on BDNF expression in hippocampus during the aging and the results demonstrated that aerobic exercise intervention in the aging process could prevent age-related hippocampal BDNF gene transcription and translation levels decrease in a certain extent. Further statistics show that the hippocampus BDNF levels were highly positively correlated to SYP. These results indicate that exercise induced-BDNF express involve in preventing aging-related hippocampal synaptic plasticity impairment and learning and memory decline.

In summary, the findings provide evidence that aerobic exercise ameliorates learning and memory deficits of aging rats induced by D-gal, which may be related to promoting hippocampal BNDF and SYP expression by aerobic exercise. However, further study is also needed to probe which BDNF signaling pathway is involved in the strengthening of synaptic plasticity and the improvement of learning and memory.

\section{References}

1. Subramaniam M, Chong SA, Vaingankar JA, et al. Prevalence of dementia in people aged 60 years and above: results from the WiSE study [J]. J Alzheimers Dis.45(4):1127-1138(2015).

2. Ritchie SJ, Dickie DA, Cox SR, et al. Brain volumetric changes and cognitive ageing during the eighth decade of life [J].Hum Brain Mapp,36(12):4910-4925(2015).

3. Łojko D, Pałys W, Czajkowska A, et al. Association of cognitive performance with the physical activity and body mass index in middle-aged and older rural inhabitants [J].Eur Rev Med Pharmacol Sci, 18(23):36453652(2014). 
4. Chang M, Jonsson PV, Snaedal J, et al. The effect of midlife physical activity on cognitive function among older adults:AGES-Reykjavik Study[J].J Gerontol A Biol Sci Med Sci,65:1369-1374 (2010).

5. Ales S. Dynamic learning and memory, synaptic plasticity and neurogenesis:an update[J].Front Behav Neurosci 8: 106(2014).

6. Yang L, Zhanhua L, Jing L, et al. Downregulation of caveolin-1 contributes to the synaptic plasticity deficit in the hippocampus of aged rats [J]. Neural Regen Res,8(29):2725-2733(2013).

7. Reddy PH, Mani G, Park BS, et al. Differential loss of synaptic proteins in Alzheimer's disease: implications for synaptic dysfunction[J].J Alzheimers Dis,7(2):103-117(2005).

8. Davies HA, Kelly A, Dhanrajan TM, et al. Synaptophysin immunogold labelling of synapses decreases in dentate gyrus of the hippocampus of aged rats [J].Brain Res, 986(1-2):191-195(2003).

9. Smith TD, Adams MM, Gallagher M, et al. Circuit-specific alterations in hippocampal synaptophysin immunoreactivity predict spatial learning impairment in aged rats [J].J Neurosci, 20(17):6587-6593(2000).

10. Cowansage KK, LeDoux JE, Monfils MH. Brain-derived neurotrophic factor:a dynamic gate keeper of neural plasticity [J].Curr Mol Pharmacol, 3:12-29(2010).

11. Edelmann E, Lessmann V, Brigadski T. Pre and postsynaptic twists in BDNF secretion and action in synaptic plasticity. Neuropharm,76 (Pt.C),610-627(2014).

12. Rothman SM, Mattson MP. Activity-dependent,tress-responsive BDNF signaling and the quest for optimal brain health and resilience throughout the lifespan [J].Neurosci,239(7):228-240 (2013).

13. Erickson KI, Gildengers AG, Butters MA. Physical activity and brain plasticity in late adulthood [J].Dialogues Clin Neurosci,15(1):99-108(2013).

14. Alomari MA, Khabour OF, Alzoubi KH, et al. Forced and voluntary exercises equally improve spatial learning and memory and hippocampal BDNF levels[J].Behav Brain Res,247:34-39 (2013).

15. Bechara R, Kelly M.Exercise improves object recognition memory and induces BDNF expression and cell proliferation in cognitively enriched rats [J].Behav Brain Res, 245:96-100(2013).

16. Liochev SI. Reactive oxygen species and the free radical theory of aging [J].Free Radic Biol, 60:1-4(2013).

17. Haider S, Liaquat L, Shahzad S, et al. A high dose of short term exogenous D-galactose administration in young male rats produces symptoms simulating the natural aging process [J]. Life Sci,3(124):110-119(2015).

18. Yu YH, Ren GP, Liu YN. Effect of FGF-21 on learning and memory ability and antioxidant capacity in brain tissue of D-galactose-induced aging mice[J].Yao Xue Xue Bao,49(7):1000-1006(2014).

19. Qiongjia Y, Jinmei Z, Wenqian D, et al. The effect of aerobic exercise on spatial learning and memory ability and expression of NCAM in hippocampus during the aging process of rats [J]. Chine Sport Science. 32(8):8590(2014).

20.D'Hooge R, De Deyn PP. Applications of the Morris water maze in the study of learning and memory[J].Brain Res Rev,36(1):60-90(2001).

21. Franklin KBJ, Paxinos G. The mouse brain in stereotaxic coordinates. San Diego: Academic Press, (1997).

22. Kenneth JL, Thomas DS. Analysis of Relative Gene Expression Data Using Real-Time Quantitative PCR and the $2^{-\Delta \Delta}$ CT Method. Methods, 25( 4):383-481(1997).

23. Hatanpää K, Isaacs KR ,Shirao T, et al. Loss of proteins regulating synaptic plasticity in normal aging of the human brain and in Alzheimer disease. J Neuropathol Exp Neurol.58(6):637-643 (1999).

24. Patterson SL. Immune dysregulation and cognitive vulnerability in the aging brain: Interactions of microglia,IL-1 $\beta$, BDNF and synaptic plasticity[J].Neuropharm.96(Pt A):11-18(2015).

25. Smith TD, Adams MM, et al. Circuit-specific alterations in hippocampal synaptophysin immunoreactivity predict spatial learning impairment in aged rats[J].J Neurosci.20 (17):6587-6593 (2000).

26. Shin MK, Jung WR, Kim HG, et al. The ganglioside GQ1b regulates BDNF expression via the NMDA receptor signaling pathway[J].Neuropharm,77:414-421(2014).

27. Hussam J, Mohamed K. Acute BDNF treatment upregulates GluR1-SAP97 and GluR2-GRIP1 interactions: implications for sustained AMPA receptor expression[J].PLOS ONE,8(2):1-13 (2013).

28. Heldt SA, Stanek L, Chhatwal, JP, et al. Hippocampus specific deletion of BDNF in adult mice impairs spatial memory and extinction of aversive memories[J].Mol.Psy,12: 656-670 (2007).

29. Petzold A, Psotta L, Brigadski T, et al. Chronic BDNF deficiency leads to an age-dependent impairment in spatial learning. Neurobiol Learn Mem.120:52-60(2015).

30.Zeng Y, Tan M, Kohyama J, et al. Epigenetic enhancement of BDNF signaling rescues synaptic plasticity in aging[J].J Neurosci,31:17800-17810(2011). 